ISSN electrónico: 2172-9077

https://doi.org/10.14201/fjc201816155170

\title{
ANÁLISIS DEL INVOLUCRAMIENTO Y LA RECONFIGURACIÓN EN YOUTUBE MÉXICO A PARTIR DEL CASO \#LADY100PESOS
}

\section{Involvement and reconfiguration analysis on YouTube Mexico from case \#lady100pesos}

\author{
Dra. Brenda Azucena Muñoz Yáñez \\ Profesor Investigador. Universidad Autónoma de Coahuila, México \\ E-mail: brenda.munoz@uadec.edu.mx \\ (iD) orcid.org/0000-0003-0962-9270
}

Fecha de recepción del artículo: 06/07/2017

Fecha de aceptación definitiva: 15/02/2018

\begin{abstract}
RESUMEN
En este artículo se analiza y discute el grado de aceptación e involucramiento que los videos de producción profesional y amateur suscitan en una plataforma como YouTube. Se toma como punto de partida el entramado semántico (Muñoz, 2014), como objeto de estudio, para explicar la manera en que los significados hegemónicos y alternativos inscritos en estos tipos de contenido se entrelazan en el acto de observar y consumir videos en dicha plataforma. Para enriquecer la discusión, se presentan resultados de investigación de un estudio de caso en YouTube México que recuperó información estadística de la interfaz del sitio para someterla a análisis de contenido. Con el registro de 364 videos principales, 3644 videos sugeridos y 161 comentarios populares, asociados el término de búsqueda \#lady100pesos, se puede observar cómo la producción de video amateur en México podría suscitar la reconfiguración de significados asociados a temas como la corrupción y la sexualización del cuerpo femenino. Se concluye que YouTube puede otorgar a sus diferentes tipos de usuarios la posibilidad de integrarse a la discusión de textos mediáticos utilizando el mismo código audiovisual que los productores profesionales de medios lo que implicaría un cambio en la manera en que los productores amateur comprenden su lugar y participación en el debate público.
\end{abstract}

Palabras clave: YouTube; medios emergentes; reconfiguración; videos amateur; entramado semántico; comunicación y género

\begin{abstract}
In this article, I analyze and discuss the acceptance and involvement degree that professional and amateur videos bring forward on YouTube. I take as a starting point the concept of semantic weaveworks (Muñoz, 2014), as an object of study, to explain the ways in which hegemonic and alternative meanings located in these kinds of content intertwine at the act of watching and consuming videos on said platform. To nurture discussion, I present research results of a case study on YouTube Mexico that retrieved YouTube's interface statistical information to apply a content analysis research tool. With the record of 364 main videos, 3644 suggested videos and the most 161 popular comments associated with the search term \#lady100pesos, I observe that amateur video production in Mexico could raise meaning reconfiguration on themes like corruption and the female body sexualization. I conclude that YouTube could give its different types of users the possibility of integrating themselves into the discussion of media texts using the same audiovisual code that professional media producers. This might imply a change in the way amateur producers understand their place and participation in the public debate.
\end{abstract}

Key words: YouTube; emergent media; reconfiguration; amateur videos; semantic weaveworks; communication and gender. 
BRENDA MUÑOZ YÁÑEZ

ANÁLISIS DEL INVOLUCRAMIENTO Y LA RECONFIGURACIÓN EN YOUTUBE MÉXICO A PARTIR DEL CASO \#LADY100PESOS

\section{INTRODUCCIÓN}

YouTube es un lugar para que la gente se involucre en nuevas formas con el video, a través de compartir, comentar y observar videos.

(YouTube, 15 de mayo de 2006, traducción propia).

Más de diez años han pasado desde que Chad Hurley, Steven Chen y Jawed Karim desplegaron las palabras con las que abrimos este artículo al tratar de describir qué es YouTube. Se recuperan aquí porque expresan el propósito original de sus fundadores, pero, además, porque ahora que el sitio se ha posicionado en el paisaje cibernético como la plataforma para la distribución de video en línea más popular del mundo (Alexa Internet, 2017), es válido preguntarse si se ha cumplido con dicho propósito. En las próximas páginas se toma un caso de estudio que fue particularmente popular en México: \#LADY100PESOS, en el que el clip de una joven en estado de ebriedad que intenta sobornar a un policía tras provocar un accidente automovilístico se viralizó y condujo a una alta producción audiovisual de tipo amateur. A partir de este caso, se examinan el grado de involucramiento y aceptación que suscitan los videos de producción profesional y no-profesional en YouTube a fin de explicar qué características de los contenidos que circulan en el sitio podrían estar suscitando el involucramiento de las audiencias con los nuevos formatos de video que circulan en YouTube.

El punto de partida de la discusión está situado en la convivencia de productos culturales producidos profesionalmente y objetos culturales no-profesionales dentro de la plataforma mencionada. En teoría, esta condición del sitio asegura una diversidad de contenidos que, como menciona Lozano (2006), contribuye a cubrir las necesidades de información, educación y entretenimiento de las audiencias. Si a esto agregamos que Jenkins, Purushotma, Clinton, Weigel y Robinson (2006) sugieren que alrededor de este tipo de plataformas se fomenta una cultura de la participación en la que los usuarios más experimentados comparten su conocimiento con los demás miembros de tal forma que puedan integrarse en el debate de la esfera cultural pública de cada sitio web, podemos advertir la relevancia de estudiar el involucramiento de las audiencias en la construcción de significados dentro de estos medios emergentes.

No obstante, haría falta realizar un par de precisiones a este cuadro en el que las audiencias se involucran con los contenidos de YouTube. En primer lugar, estudios anteriores (Muñoz, 2012; Burgess y Green, 2009; Lang, 2007) han encontrado que al interior de la comunidad de YouTube existe una compleja jerarquización de usuarios con base en su grado de profesionalización y popularidad; y que, además de las retribuciones sociales que reciben los usuarios del sitio, su participación también puede verse recompensada monetariamente, lo que ubicaría, al menos, a una parte de los usuarios más prominentes, como productores semi-profesionales. Es decir que, examinar las maneras en que se vinculan los videos profesionales y no-profesionales en la interfaz de YouTube, podría indicar también si las características de esta plataforma funcionan a favor o en contra de comunidades fundadas en la cultura de la participación ya que, a pesar de la diversidad de contenidos que se pueden encontrar en el sitio, no debemos soslayar la injerencia que tienen las corporaciones de medios al interior de la comunidad ni descartar la posibilidad de que los usuarios más prominentes, en aras de proteger su ingreso económico, incurran en prácticas que más que alentar la participación de todos los usuarios la restrinjan.

En el siguiente apartado, se explica la propuesta teórico-metodológica utilizada para estudiar YouTube y lidiar con estas complicaciones delineadas. Posteriormente, se exponen los resultados de investigación de un estudio de caso en YouTube México que fue considerado relevante para discutir el involucramiento y la aceptación de los contenidos audiovisuales de origen amateur que circulan en el

Ediciones Universidad de Salamanca / CC BY - NC ND Fonseca, Journal of Communication, n. 16, 2018, pp. 155-170 
sitio. Finalmente, se exponen las conclusiones respecto al lugar que ocupa la participación que tienen los usuarios del sitio en el debate público. En este punto, es importante comentar que este estudio se lleva a cabo en el Cuerpo Académico «Comunicación para el desarrollo social» de la Universidad Autónoma de Coahuila, que cuenta con financiamiento del «Programa para el Desarrollo Profesional Docente» de la Secretaría de Educación Pública de México y tiene por objetivo examinar las maneras en que se vinculan los videos amateur con los profesionales en el entramado semántico de YouTube México, y cómo es que los significados hegemónicos y alternativos que emergen de ellos son reconfigurados.

\section{Propuesta Teórico-MetodológiCA: ENTRAMAdo SEMÁNTiCo}

En otras ocasiones (Muñoz, 2016; 2014; Muñoz y Vélez, 2016; Muñoz y Corona, 2014), se ha utilizado el concepto de entramado semántico para explicar la manera en que la producción profesional y amateur se integran en un solo flujo de videos de YouTube. Teóricamente, dicho concepto se nutre, por un lado, de la consideración de la cultura como un proceso de absorción, adaptación e innovación de significados que refleja las influencias multidireccionales entre distintos grupos sociales (Morris, 2002; García Canclini, 1997). Por el otro lado, está inspirado en la invitación de los estudios culturales a observar cómo se entrelazan los discursos hegemónicos y alternativos en el acto de ver televisión. John Fiske (1992) explica los conflictos y luchas involucradas en dicho acto como un maelstrom semántico en el que las distintas significaciones de la audiencia se integran como pequeños caudales de agua a un vórtice. La interpretación que la audiencia realiza sobre un producto mediático se vería afectada por las ideas preconcebidas de cada receptor de tal forma que la corriente principal del significado de un texto audiovisual es desviada por las corrientes divergentes y contracorrientes que surgen en el momento de la recepción. En este sentido, YouTube constituye una arena de lucha por el significado en el que grupos de productores y consumidores de medios participan y se influyen unos a otros a través de las distintas características de la interfaz.

No obstante, a diferencia de la desorganización que sugiere el término maelstrom, en YouTube se reconoce una estructura subyacente, y es en este punto donde el concepto de entramado semántico funciona también como una propuesta metodológica. Si se considera que los videos producidos profesionalmente forman una corriente principal de significado que es trasgredida por la circulación de otros videos producidos de forma amateur por los receptores de dicho texto mediático, tenemos que para estudiar el entramado semántico debemos analizar los distintos niveles en que se entrelazan estas dos corrientes: interfaz y contenidos. En el primer nivel, estudiar la interfaz del sitio permite conocer qué características y funcionalidades de la plataforma aseguran la diversidad de contenidos. Por su lado, el análisis de contenidos ofrece la posibilidad de indagar en los procesos de reconfiguración de significados que dicha diversidad de contenidos podría estar propiciando. De ahí que se defina el entramado semántico de YouTube como la red estructural de videos profesionales y amateur que se encuentran agrupados en torno a una palabra o tema y a través de la cual los diferentes discursos audiovisuales de los usuarios de YouTube interactúan, unos con mayor fuerza que otros, en la construcción del significado de textos mediáticos.

Tomando en cuenta lo anterior, se realizó un diseño metodológico orientado hacia el paradigma cuantitativo y la perspectiva de estudio de caso. Para examinar la distribución de los contenidos profesionales y amateur, es decir el nivel de la interfaz, se diseñó en lenguaje Python un programa de recolección de información y metadatos mediante la API de YouTube. La Application Programming Interface es una interfaz programática diseñada para realizar operaciones sistemáticas o responder a peticiones de información a través de la web. Específicamente, la API de YouTube consiste en una serie

Ediciones Universidad de Salamanca / CC BY - NC ND Fonseca, Journal of Communication, n. 16, 2018, pp. 155-170 
de recursos que Google, Inc. pone a disponibilidad del público con la intención de facilitar a programadores y terceros el diseño de aplicaciones para interactuar con el sitio (Google Developers, 2016). Dicho programa recuperó datos estadísticos y metadatos para los videos arrojados por un término de búsqueda en el sitio. Posteriormente, se agregó a esta base de datos la información de los canales de los cuales provienen dichos videos y las listas de videos relacionados para cada uno de los videos principales. Así se conformó una base de datos de 364 videos principales y 3644 videos directamente asociados a ellos por el algoritmo de búsqueda de YouTube.

Para estudiar el nivel de los contenidos, se elaboró un instrumento de análisis de contenido cuantitativo que tuvo como unidad de análisis el clip audiovisual y buscó medir tanto aspectos formales de los 364 videos principales como valoraciones de los temas implicados en el caso de estudio. Para diferenciar las distintas corrientes de significado que fluyen a través de YouTube, se recuperó y actualizó la clasificación que realiza Fiske (1999) sobre los textos televisivos: texto primario, que corresponde al mensaje original; texto secundario, que se refiere a las producciones de la industria mediática y publicitaria que fortalecen el significado de un texto primario; y texto terciario, aquel que se produce a partir de la interpretación que la audiencia hace del texto primario.

A esta clasificación de Fiske (1999), se agregó un cuarto tipo de texto bajo la denominación «Paratexto». En esta categoría se agruparon todos aquellos clips de video que, si bien acompañan y refuerzan el significado del texto original, no se refieren directamente a dicho texto primario. En el flujo de videos de YouTube, pueden identificarse estos cuatro tipos de texto, aunque ciertamente, como sucedió en el caso que aquí examinamos, el origen del texto primario no siempre corresponde a contenido producido profesionalmente. En el siguiente apartado comentamos los criterios que seguimos para la selección del caso de estudio.

Una vez conformada la base de datos, los metadatos de cada clip fueron utilizados también para determinar el grado de aceptación de cada video y el nivel de involucramiento que propician. De igual forma, la información de los canales fue utilizada para determinar el tipo de usuario que cargó cada video. Para determinar los distintos tipos de usuarios se aplicaron criterios para distinguir aquellos identificados por Lang (2007):

Los antiguos participantes ya no suben videos, pero mantienen una cuenta, observan videos en el sitio y realizan comentarios ocasionales. Los usuarios casuales, quienes típicamente no tienen una cuenta, tienden a ver videos cuando desean buscar algo específico, cuando navegan en el sitio o cuando alguien los insta con un link a un video particular. Los participantes activos tienen una cuenta y usualmente suben videos o al menos participan dejando comentarios en la página del video o canal de otras personas. (...) Los youtubers, o «Tubers» son gente que tienen un involucramiento más intenso con YouTube en términos de la cantidad y tipo de participación. (...) El grupo final, celebridades de YouTube, comparten cualidades similares a los youtubers. Sin embargo, ellos son bien conocidos en el sitio y frecuentemente fuera de él. (Lang, 2007, p. 5, traducción propia, énfasis añadido).

A esta categorización de usuarios, se añadió el tipo «Medios profesionales», los cuales se caracterizan por una alta producción audiovisual y popularidad. Los datos fueron procesados mediante el programa estadísticos IBM-SPSS y los resultados pertinentes a la discusión sobre involucramiento y reconfiguración se presentan y discuten más adelante. 


\section{SELECCIÓN DEL CASO DE ESTUdio}

Como ya se mencionó, la propuesta es que al interior de los clústeres de videos en YouTube es posible identificar una estructura que organiza los diversos clips, presumiblemente de acuerdo con una jerarquía de usuarios. De ser así, esto significaría que la infraestructura del sitio incide en la manera en que se presenta la diversidad de contenidos, es decir, la manera en que los contenidos hegemónicos se vinculan con contenidos alternativos propiciando una negociación de significados sobre los distintos temas que se discuten en la esfera cultural pública del sitio web. Por este motivo, fue indispensable seleccionar un término de búsqueda que pudiera ayudarnos a explicar cómo se relacionan los videos producidos por los distintos tipos de usuarios entre sí. Para escoger el término de búsqueda, se realizó una exploración en YouTube México que involucró la identificación de los temas más discutidos por los usuarios de México con mayor número de suscriptores y reproducciones.

En primera instancia se obtuvo una lista con los 25 usuarios del sitio más populares de acuerdo con los criterios arriba expuestos para obtener una muestra de videos que fueron sometidos a análisis de contenido. Este primer acercamiento permitió identificar que los asuntos que más discuten los youtubers mexicanos están relacionados con el tema de salud, género y sexualidad (Muñoz, Maldonado y Vélez, 2016). De ahí que se hicieran pruebas con distintos términos de búsqueda para identificar un asunto que fuera relevante y actual para la comunidad de YouTube México. Uno de estos términos fue \#lady100pesos, el cual fue finalmente seleccionado porque logró reunir una alta producción nacional en pocos meses y, además, incluyó la participación de medios profesionales en el clúster.

\#lady100pesos es el seudónimo con el que se identificó a una joven de 18 años que en enero de 2016 protagonizó un accidente automovilístico en la ciudad de Guanajuato, México. Luego del accidente, un testigo registró en video la interacción que sostuvo la joven con elementos de la policía del estado quienes la detuvieron por conducir en estado de ebriedad y alterar el orden público. El seudónimo hace referencia a la pretensión de la joven por sobornar a los policías con un billete de cien pesos (Informador, 22 de enero de 2016). El video original fue cargado en la página de un usuario privado de Facebook y fue posteriormente difundido por medios locales de distintas ciudades del país. Pero no fue sino hasta unos meses después que comenzaron a cargarse distintos tipos de video en YouTube y tanto el clip de video como la figura misma de la mujer alcanzaron un alto grado de popularidad en diferentes redes sociales donde se discutían los pormenores de la interacción en términos de los atributos físicos de la protagonista, su comportamiento y representación de la clase alta mexicana. Además, este es un caso representativo de un fenómeno que ha ido ocurriendo con frecuencia en la esfera pública digital mexicana en el que los usuarios de medios emergentes crean distintos tipos de contenidos que ridiculizan el comportamiento de mujeres de la clase alta en México (Reina, 29 de abril de 2016).

Por otro lado, en un estudio anterior (Muñoz y Corona, 2014) que implementó la estrategia teórico-metodológica que aquí se propone, se detectó que, en el caso de un texto primario producido profesionalmente por un medio de comunicación tradicional como el cine, los videos profesionales gozan de mayor movilidad en la interfaz del sitio. De tal forma que la selección de un término de búsqueda cuyo texto primario corresponde a contenido amateur podría ayudar a comprobar la presencia o ausencia de esta característica de la interfaz de YouTube.

\section{RESULTADOS}

El primer punto que se recupera para discutir el involucramiento y aceptación de los videos analizados es el espectro de influencia que \#lady100pesos tuvo en YouTube. Si bien el video original titulado «Lady 100 pesos o Lady Tepetapa» fue publicado en enero de 2016 a través de Facebook, en la

Ediciones Universidad de Salamanca / CC BY - NC ND Fonseca, Journal of Communication, n. 16, 2018, pp. 155-170 
Gráfica 1 podemos apreciar que su espectro de influencia en YouTube se extendió por al menos seis meses, lapso en que fueron cargados los 364 videos principales asociados al término de búsqueda. Esto podría sugerir que el involucramiento con contenidos amateur tiende a aumentar a medida que el tema es discutido en distintas plataformas mediáticas con una posible implicación de que las esferas culturales públicas de los sitios web con frecuencia convergen.

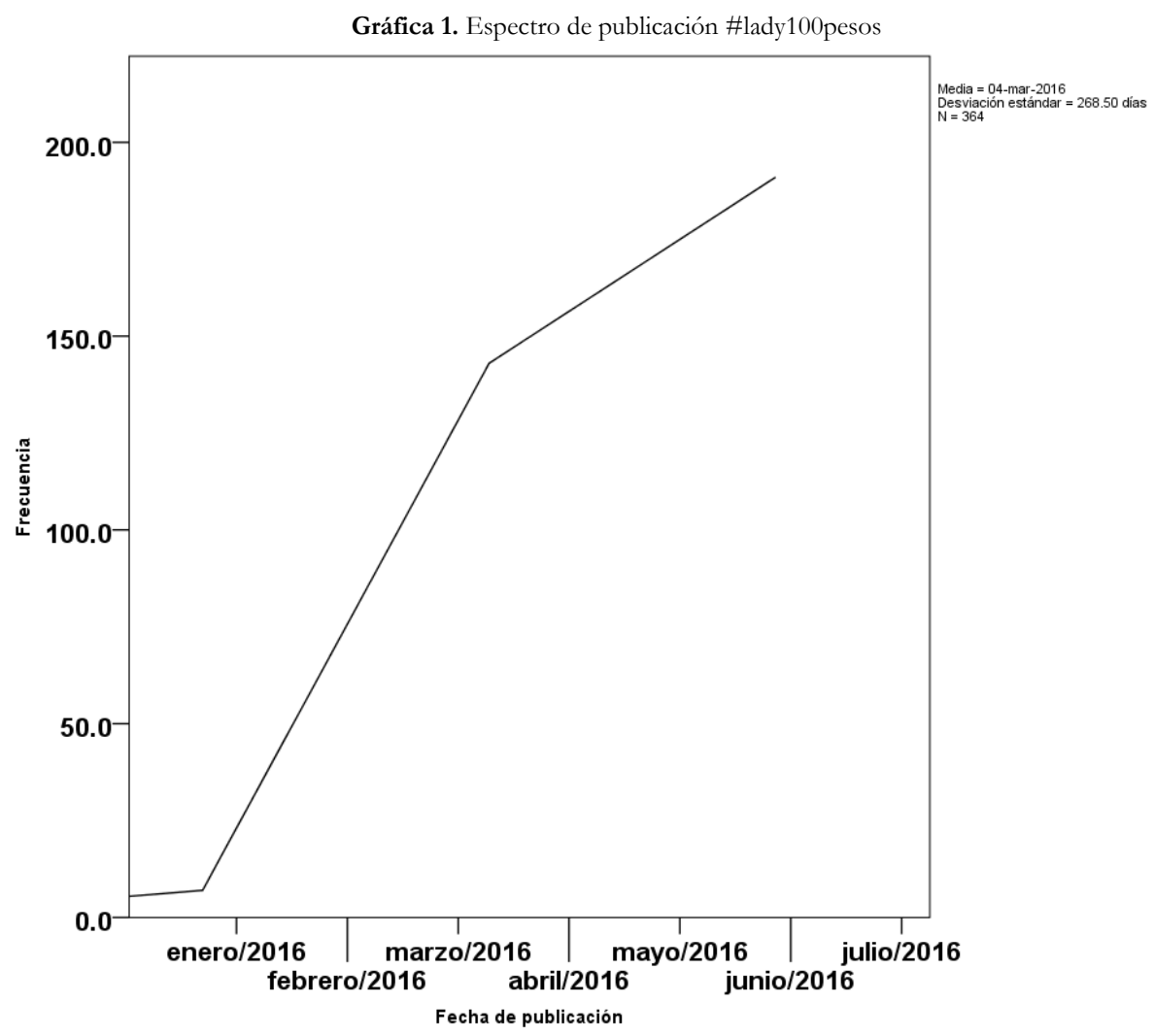

Elaboración propia.

Los videos principales recuperados tuvieron una duración media de 452,60 segundos y en promedio contaron con 385.972 reproducciones y 332 comentarios. Con estos datos se puede apreciar una disparidad entre las distintas formas en que las audiencias se involucran con los contenidos audiovisuales. De ahí que se consideraran estas características para medir el nivel de involucramiento que propician los contenidos; para medir este aspecto se contrastó la suma del total de comentarios de cada video, los votos a favor (Likes) o en contra (Dislikes) que tuvieren con el total de reproducciones. De igual forma, para medir el grado de aceptación de los clips se contrastó el número de votos a favor (Likes) con el total de votos de cada video. En términos generales, la muestra de clips analizada obtuvo un porcentaje de involucramiento de 1,99 \% y un porcentaje de aceptación de 69,17\%. Esto parece indicar que es bajo el uso de las características de la plataforma que ofrecen al usuario nuevas formas de participación en el consumo de objetos audiovisuales, sobre todo de aquellas donde se requiere un involucramiento activo de las audiencias como es el caso de la sección de comentarios. 
Como el propósito es comprender la aceptación y el involucramiento diferenciado, se contrastaron estas dos variables con los tipos de usuarios de YouTube a fin de detectar si alguno de ellos se asocia con un grado mayor en estas categorías. Es importante anotar que no se detectó la presencia de antiguos participantes y, como era de esperarse, la mayoría del contenido analizado fue cargado en el sitio web por usuarios activos $(33,4 \%)$ y youtubers $(31 \%)$. No obstante, al contrastar el nivel de aceptación y grado de involucramiento que generan los videos cargados por los diferentes tipos de usuarios, se puede observar una relación positiva ascendente entre el grado de profesionalización del usuario y el porcentaje de aceptación e involucramiento de las audiencias: las celebridades de YouTube tienen un porcentaje de aceptación mayor, $84,43 \%$, que el del resto de los usuarios; y por otro lado, el contenido que suscita mayor involucramiento es aquel producido por medios profesionales, $4,79 \%$ (Tabla 1). Esto podría sugerir que las audiencias de los videos principales tienden a observar de una manera más crítica los contenidos producidos profesionalmente ya que comentan estos contenidos.

Tabla 1. Involucramiento y aceptación por tipo de usuario

\begin{tabular}{|c|c|c|c|}
\hline Tipo de usuario & & $\begin{array}{c}\text { Porcentaje de } \\
\text { involucramiento } \\
\text { con video } \\
\text { principal }\end{array}$ & $\begin{array}{l}\text { Porcentaje de } \\
\text { Aceptación de } \\
\text { video principal }\end{array}$ \\
\hline \multirow[t]{3}{*}{ Usuario Casual } & Media & .9777 & 54.1145 \\
\hline & $\mathrm{N}$ & 38 & 38 \\
\hline & $\mathrm{DE}$ & 2.41325 & 41.19114 \\
\hline \multirow[t]{3}{*}{ Usuario Activo } & Media & 2.9260 & 65.0378 \\
\hline & $\mathrm{N}$ & 122 & 122 \\
\hline & $\mathrm{DE}$ & 5.44991 & 35.38391 \\
\hline \multirow[t]{3}{*}{ YouTuber } & Media & 1.1323 & 68.6177 \\
\hline & $\mathrm{N}$ & 113 & 113 \\
\hline & $\mathrm{DE}$ & 1.91825 & 33.73686 \\
\hline \multirow[t]{3}{*}{ Celebridad de YouTube } & Media & 1.6578 & $\underline{84.4261}$ \\
\hline & $\mathrm{N}$ & 74 & 74 \\
\hline & DE & 2.10470 & 17.63415 \\
\hline \multirow[t]{3}{*}{ Medios profesionales } & Media & $\underline{4.7853}$ & 69.8002 \\
\hline & $\mathrm{N}$ & 17 & 17 \\
\hline & $\mathrm{DE}$ & 13.39501 & 35.99476 \\
\hline \multirow[t]{3}{*}{ Total } & Media & 1.9948 & 69.1728 \\
\hline & $\mathrm{N}$ & 364 & 364 \\
\hline & DE & 4.63120 & 33.73613 \\
\hline
\end{tabular}

Elaboración propia.

Otros aspectos medidos y que podrían estar asociados con un mayor grado de aceptación e involucramiento fue el tipo de texto, así como el tipo de producción y formato del clip. En la Tabla 2 se observa que el texto secundario está asociado a un porcentaje más alto de aceptación $(78,88 \%$ ) y el 
paratexto a un porcentaje de involucramiento mayor (3,46\%). De igual forma en la Tabla 3, se aprecia que el tipo de producción live action está asociado con un alto porcentaje de involucramiento $(4,96)$ y aceptación (76,08 \%). Finalmente, respecto al formato del clip (Tabla 4), los playthrough, grabaciones en vivo de la experiencia de juego del productor del video, destacan por asociarse con un porcentaje alto de involucramiento $(12,24 \%)$ y aceptación $(85,93 \%)$.

Tabla 2. Involucramiento y aceptación por tipo de texto

\begin{tabular}{|c|c|c|c|}
\hline Tipo de Texto & & $\begin{array}{l}\text { Porcentaje de involucramiento } \\
\text { con video principal }\end{array}$ & $\begin{array}{c}\text { Porcentaje de Aceptación } \\
\text { de video principal }\end{array}$ \\
\hline \multirow[t]{3}{*}{ Texto Primario } & Media & .6274 & 66.5984 \\
\hline & $\mathrm{N}$ & 33 & 33 \\
\hline & $\mathrm{DE}$ & 1.17534 & 38.08931 \\
\hline \multirow[t]{3}{*}{ Texto Secundario } & Media & .9649 & $\underline{78.8849}$ \\
\hline & $\mathrm{N}$ & 55 & 55 \\
\hline & $\mathrm{DE}$ & 1.22798 & 22.75513 \\
\hline \multirow{3}{*}{ Texto Terciario } & Media & 2.4977 & 67.2496 \\
\hline & $\mathrm{N}$ & 182 & 182 \\
\hline & $\mathrm{DE}$ & 4.72470 & 33.03427 \\
\hline \multirow{3}{*}{ Paratexto } & Media & $\underline{3.4602}$ & 69.8596 \\
\hline & $\mathrm{N}$ & 39 & 39 \\
\hline & $\mathrm{DE}$ & 9.10515 & 33.93003 \\
\hline \multirow[t]{4}{*}{ Total } & Media & 2.1466 & 69.5805 \\
\hline & $\mathrm{N}$ & 309 & 309 \\
\hline & $\mathrm{DE}$ & 4.95404 & 32.31838 \\
\hline & Casos perdidos & 55 & 55 \\
\hline
\end{tabular}

Elaboración propia.

Tabla 3. Involucramiento y aceptación por tipo de producción

\begin{tabular}{|c|c|c|c|}
\hline Tipo de producción & & $\begin{array}{l}\text { Porcentaje de involucramiento } \\
\text { con video principal }\end{array}$ & $\begin{array}{l}\text { Porcentaje de Aceptación } \\
\text { de video principal }\end{array}$ \\
\hline \multirow{3}{*}{ Recut } & Media & .9045 & 75.1359 \\
\hline & $\mathrm{N}$ & 58 & 58 \\
\hline & $\mathrm{DE}$ & 1.70315 & 31.02397 \\
\hline \multirow[t]{3}{*}{ Mashup } & Media & .8861 & 64.2989 \\
\hline & $\mathrm{N}$ & 98 & 98 \\
\hline & $\mathrm{DE}$ & 1.22563 & 34.89605 \\
\hline \multirow[t]{3}{*}{ Animación } & Media & 2.9504 & 68.5262 \\
\hline & $\mathrm{N}$ & 7 & 7 \\
\hline & $\mathrm{DE}$ & 4.21633 & 36.29929 \\
\hline \multirow[t]{3}{*}{ Live action } & Media & $\underline{4.9627}$ & $\underline{76.0886}$ \\
\hline & $\mathrm{N}$ & 96 & 96 \\
\hline & $\mathrm{DE}$ & 7.69114 & 25.54864 \\
\hline \multirow[t]{3}{*}{ Grabación casual } & Media & 1.2204 & 66.9634 \\
\hline & $\mathrm{N}$ & 55 & 55 \\
\hline & $\mathrm{DE}$ & 3.12581 & 39.59383 \\
\hline \multirow[t]{4}{*}{ Total } & Media & 2.2404 & 70.4661 \\
\hline & $\mathrm{N}$ & 314 & 314 \\
\hline & $\mathrm{DE}$ & 4.93420 & 32.77768 \\
\hline & Casos perdidos & 50 & 50 \\
\hline
\end{tabular}

Elaboración propia. 
Respecto a estos resultados, es importante considerar que el playtrough junto con el $v \log (6,25 \%$ de involucramiento y 77,65 \% de aceptación) y el sketch (2,58 \% de involucramiento y 79,56\% de aceptación) son formatos que típicamente requieren grabación de acción en vivo, por lo que se podría aventurar que el tipo de producción tiene una mayor incidencia en el involucramiento y aceptación de las audiencias. El vlog es el formato emblema de YouTube y se refiere a una serie de clips de video ordenados cronológicamente; formalmente se distingue por presentar a cuadro al productor del video como una cabeza parlante a través de una cámara web. El sketch, por su cuenta, se refiere a una escena cómica de corta duración realizada por actores o comediantes.

Tabla 4. Involucramiento y aceptación por formato del video

\begin{tabular}{|c|c|c|c|}
\hline \multicolumn{2}{|c|}{ Formato del video } & $\begin{array}{l}\text { Porcentaje de involucramiento } \\
\text { con video principal }\end{array}$ & $\begin{array}{c}\text { Porcentaje de Aceptación } \\
\text { de video principal }\end{array}$ \\
\hline \multirow[t]{3}{*}{ Vlog } & Media & $\underline{6.2670}$ & 77.6537 \\
\hline & $\mathrm{N}$ & 64 & 64 \\
\hline & $\mathrm{DE}$ & 7.90220 & 22.75504 \\
\hline \multirow[t]{3}{*}{ Sketch } & Media & $\underline{2.5830}$ & $\underline{79.5698}$ \\
\hline & $\mathrm{N}$ & 8 & 8 \\
\hline & $\mathrm{DE}$ & 1.52575 & 17.46883 \\
\hline \multirow{3}{*}{$\begin{array}{l}\text { Fanvid o } \\
\text { AMV }\end{array}$} & Media & 1.1177 & 60.2543 \\
\hline & $\mathrm{N}$ & 15 & 15 \\
\hline & $\mathrm{DE}$ & 1.71976 & 38.78323 \\
\hline \multirow[t]{3}{*}{ Compilación } & Media & .7514 & 60.1056 \\
\hline & $\mathrm{N}$ & 50 & 50 \\
\hline & $\mathrm{DE}$ & 1.05341 & 37.46025 \\
\hline \multirow[t]{3}{*}{ Newscast } & Media & .7682 & 71.8775 \\
\hline & $\mathrm{N}$ & 78 & 78 \\
\hline & $\mathrm{DE}$ & .78417 & 29.98054 \\
\hline \multirow[t]{3}{*}{ Documento } & Media & 1.0509 & 66.7936 \\
\hline & $\mathrm{N}$ & 64 & 64 \\
\hline & $\mathrm{DE}$ & 1.66584 & 39.32493 \\
\hline \multirow[t]{3}{*}{ Filk } & Media & 1.3215 & 72.6204 \\
\hline & $\mathrm{N}$ & 24 & 24 \\
\hline & $\mathrm{DE}$ & 2.80730 & 35.79251 \\
\hline \multirow[t]{3}{*}{ Playthrough } & Media & $\underline{12.2467}$ & $\underline{85.9366}$ \\
\hline & $\mathrm{N}$ & 5 & 5 \\
\hline & $\mathrm{DE}$ & 17.35858 & 18.37903 \\
\hline \multirow[t]{4}{*}{ Total } & Media & 2.2604 & 70.0302 \\
\hline & $\mathrm{N}$ & 308 & 308 \\
\hline & $\mathrm{DE}$ & 4.97668 & 33.04310 \\
\hline & Casos perdidos & 56 & 56 \\
\hline
\end{tabular}

Elaboración propia.

Por otro lado, para inspeccionar con mayor detalle la apreciación crítica que realizan las audiencias e indagar en los mecanismos de reconfiguración, se examinó la sección de comentarios. La interfaz de YouTube ofrece la opción de interactuar mediante comentarios, y en ese sentido, destaca que sólo el $1.6 \%$ de los usuarios que cargaron los videos principales contenidos en la muestra participaron también en el hilo de comentarios de sus videos. Destaca porque sugiere que la sección de comenta- 
rios es un espacio para que las audiencias interactúen entre sí y no precisamente para interactuar con los productores de contenidos.

Tabla 5. Contenido del comentario por tipo de usuario

\begin{tabular}{|c|c|c|c|}
\hline \multirow[b]{2}{*}{ Tipo de usuario } & \multicolumn{2}{|c|}{$\begin{array}{c}\text { Contenido del comentario } \\
\text { principal }\end{array}$} & \multirow[t]{2}{*}{ Total } \\
\hline & Aprobación & $\begin{array}{l}\text { Sugerencia o } \\
\text { crítica }\end{array}$ & \\
\hline \multirow[t]{2}{*}{ Usuario Casual } & 2 & 8 & 10 \\
\hline & $20.0 \%$ & $80.0 \%$ & $100.0 \%$ \\
\hline \multirow[t]{2}{*}{ Usuario Activo } & 18 & 32 & 50 \\
\hline & $36.0 \%$ & $64.0 \%$ & $100.0 \%$ \\
\hline \multirow[t]{2}{*}{ YouTuber } & 15 & 36 & 51 \\
\hline & $29.4 \%$ & $70.6 \%$ & $100.0 \%$ \\
\hline \multirow{2}{*}{$\begin{array}{l}\text { Celebridad de } \\
\text { YouTube }\end{array}$} & 14 & 30 & 44 \\
\hline & $31.8 \%$ & $68.2 \%$ & $100.0 \%$ \\
\hline \multirow[t]{2}{*}{ Medios profesionales } & 3 & 3 & 6 \\
\hline & $50.0 \%$ & $50.0 \%$ & $100.0 \%$ \\
\hline \multirow[t]{2}{*}{ Total } & 52 & 109 & 161 \\
\hline & $32.3 \%$ & $67.7 \%$ & $100.0 \%$ \\
\hline
\end{tabular}

Elaboración propia.

En esta sección predominaron los comentarios de tipo declaración, con un tono llano o plano y contenido orientado hacia la sugerencia o crítica de temas relacionados al video principal. Además, fue relevante detectar que los comentarios orientados hacia la sugerencia o crítica de contenidos fueron más frecuentes en los videos cargados por usuarios activos, youtubers y celebridades (Tabla 5). Esto matiza la calidad del involucramiento que suscitan los videos producidos profesionalmente, ya que exhiben un bajo recuento de comentarios en esta categoría.

En cuanto a la sección de videos relacionados o lista de sugerencias, se presenta una gráfica de ruta (Gráfica 2) que explica visualmente cómo viajan y se relacionan entre sí los contenidos profesionales y amateur sobre \#lady100pesos en YouTube Méxito. Para elaborarla, se contrastó la relación entre los tipos de usuarios de los videos principales y los tipos de usuarios de los videos que aparecieron en la lista de sugerencias. Se puede observar que los distintos tipos de usuarios se relacionan entre sí, con excepción de los usuarios casuales y los medios profesionales que tienden a vincularse principalmente con contenidos producidos por celebridades (Tabla 6). No obstante, se aprecia una ligera tendencia a 
reunir todas las corrientes y caminos para discutir el tema \#lady100pesos en el centro, representado típicamente por youtubers y celebridades.

Tabla 6. Movilidad y visibilidad de contenidos en videos relacionados

\begin{tabular}{|c|c|c|c|c|c|c|}
\hline \multirow[b]{2}{*}{$\begin{array}{c}\text { Tipo de } \\
\text { usuario de } \\
\text { video principal }\end{array}$} & \multicolumn{5}{|c|}{ Tipo de usuario de videos relacionados } & \multirow[b]{2}{*}{ Total } \\
\hline & Usuario Casual & $\begin{array}{l}\text { Usuario } \\
\text { Activo }\end{array}$ & YouTuber & $\begin{array}{l}\text { Celebridad de } \\
\text { YouTube }\end{array}$ & $\begin{array}{c}\text { Medios } \\
\text { profesionales }\end{array}$ & \\
\hline \multirow[t]{2}{*}{ Usuario Casual } & 20 & 82 & 117 & $\underline{142}$ & 19 & 380 \\
\hline & $5.2 \%$ & $21.6 \%$ & $30.8 \%$ & $37.4 \%$ & $5.0 \%$ & $100 \%$ \\
\hline \multirow[t]{2}{*}{ Usuario Activo } & 50 & $\underline{440}$ & 331 & 341 & 56 & 1218 \\
\hline & $4.1 \%$ & $36.1 \%$ & $27.2 \%$ & $28.0 \%$ & $4.6 \%$ & $100 \%$ \\
\hline \multirow[t]{2}{*}{ YouTuber } & 38 & 189 & $\underline{456}$ & 388 & 66 & 1137 \\
\hline & $3.4 \%$ & $16.6 \%$ & $40.1 \%$ & $34.1 \%$ & $5.8 \%$ & $100 \%$ \\
\hline \multirow{2}{*}{$\begin{array}{l}\text { Celebridad de } \\
\text { YouTube }\end{array}$} & 18 & 105 & 182 & $\underline{392}$ & 42 & 739 \\
\hline & $2.5 \%$ & $14.2 \%$ & $24.6 \%$ & $53.0 \%$ & $5.7 \%$ & $100 \%$ \\
\hline \multirow{2}{*}{$\begin{array}{l}\text { Medios } \\
\text { profesionales }\end{array}$} & 2 & 26 & 52 & $\underline{62}$ & 28 & 170 \\
\hline & $1.1 \%$ & $15.3 \%$ & $30.6 \%$ & $36.5 \%$ & $16.5 \%$ & $100 \%$ \\
\hline \multirow[t]{2}{*}{ Total } & 128 & 842 & 1138 & 1325 & 211 & 3644 \\
\hline & $3.5 \%$ & $23.1 \%$ & $31.2 \%$ & $36.4 \%$ & $5.8 \%$ & $100 \%$ \\
\hline
\end{tabular}

$\mathrm{X}^{2}=313.960, \mathrm{p}=.000$ Elaboración propia.

En lo que se refiere a la examinación de los contenidos de los clips, se ha mencionado que la aceptación e involucramiento podrían estar relacionados con aspectos formales como el tipo de producción y formato. Sin embargo, se observaron también cómo se relacionan dichas categorías con contenidos que actualizan y reconfiguran los significados vinculados con el caso de estudio. En primer lugar, destaca que en cuanto a la forma en que los clips analizados hacen referencia al evento original, la grabación de acción planeada en vivo (live action), tipo de producción con mayores niveles de involucramiento, presentó principalmente una referencia de tipo collage $(31,2 \%)$. Se clasificó este tipo de referencia como aquella que tiene el propósito de «criticar, retar y posiblemente sublevarse al poder de las imágenes producidas, y distribuidas, por las corporaciones mediáticas» (Wees, 1993, p. 33). 
BRENDA MUÑOZ YÁÑEZ

ANÁLISIS DEL INVOLUCRAMIENTO Y LA RECONFIGURACIÓN EN YOUTUBE MÉXICO A PARTIR DEL

CASO \#LADY100PESOS

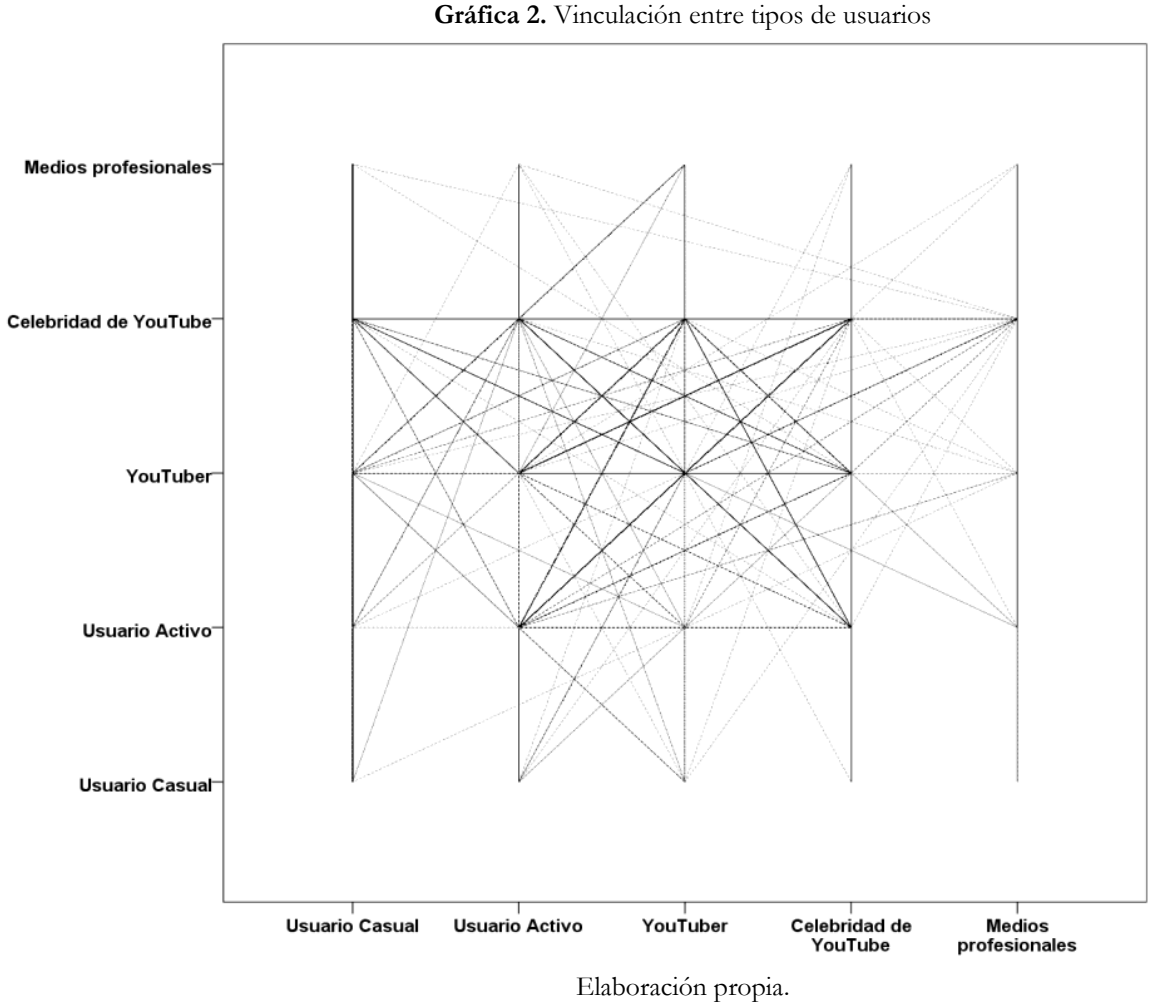

Tabla 7. Referencia por formato del video

\begin{tabular}{|c|c|c|c|}
\hline \multirow{2}{*}{$\begin{array}{l}\text { Formato del } \\
\text { video }\end{array}$} & \multicolumn{2}{|c|}{ Referencia } & \multirow[t]{2}{*}{ Total } \\
\hline & $\begin{array}{c}\text { Homenaje, Pastiche } \\
\text { y Alusión }\end{array}$ & $\begin{array}{c}\text { Collage, Parodia y } \\
\text { Apropiación }\end{array}$ & \\
\hline \multirow[t]{2}{*}{ Vlog } & 23 & 31 & 54 \\
\hline & $42.6 \%$ & $57.4 \%$ & $100.0 \%$ \\
\hline \multirow[t]{2}{*}{ Sketch } & 3 & 4 & 7 \\
\hline & $42.9 \%$ & $57.1 \%$ & $100.0 \%$ \\
\hline \multirow[t]{2}{*}{ Fanvid o AMV } & 10 & 5 & 15 \\
\hline & $66.7 \%$ & $33.3 \%$ & $100.0 \%$ \\
\hline \multirow[t]{2}{*}{ Compilación } & 33 & 10 & 43 \\
\hline & $76.7 \%$ & $23.3 \%$ & $100.0 \%$ \\
\hline \multirow{2}{*}{ Newscast } & 37 & 32 & 69 \\
\hline & $53.6 \%$ & $46.4 \%$ & $100.0 \%$ \\
\hline \multirow{2}{*}{ Documento } & 10 & 14 & 24 \\
\hline & $41.7 \%$ & $58.3 \%$ & $100.0 \%$ \\
\hline \multirow[t]{2}{*}{ Filk } & 17 & 7 & 24 \\
\hline & $70.8 \%$ & $29.2 \%$ & $100.0 \%$ \\
\hline \multirow[t]{2}{*}{ Playthrough } & 3 & 2 & 5 \\
\hline & $60.0 \%$ & $40.0 \%$ & $100.0 \%$ \\
\hline \multirow[t]{2}{*}{ Total } & 136 & 105 & 241 \\
\hline & $56.4 \%$ & $43.6 \%$ & $100.0 \%$ \\
\hline $\begin{array}{l}\text { Casos } \\
\text { perdidos }\end{array}$ & & & 123 \\
\hline
\end{tabular}

$\mathrm{X}^{2}=16.986, \mathrm{p}=0.17$ Elaboración propia. 
En cuanto al tipo de referencia en relación con el formato, en la Tabla 7 puede observarse que el $v \log$ estuvo principalmente asociado con referencias de tipo collage, parodia y apropiación (57,4 \%), las cuales se distinguen por proponer un cambio en la interpretación del texto original. En contraposición, formatos como la compilación y el filk mostraron una tendencia a asociarse con referencias de tipo homenaje, pastiche y alusión, las cuales se distinguen por celebrar el texto original. De igual forma, sobresale el caso de los newscasts, pequeñas grabaciones realizadas por usuarios no profesionales que simulan el estilo y formato de los noticieros tradicionales. Aunque estos clips no destacaron por su nivel de involucramiento, se puede apreciar que su presencia fue importante en la muestra y resultaron asociados principalmente al homenaje, pastiche y alusión.

Conviene aclarar que desde que fue seleccionado el caso de estudio se prefiguraron los temas que podrían estar inmiscuidos en el evento de \#lady100pesos y que son continuamente discutidos en la esfera cultural pública de YouTube: género y sexualidad y características de la clase alta en México. Es relevante mencionar que solo el 42,5\% de los clips de video desplegaron un comentario directo del presentador (anchor) sobre el caso analizado. En ese sentido, la incidencia de valoración de los distintos temas se presentó reducida.

Al realizar valoraciones sobre la belleza física e indumentaria de la protagonista del evento \#lady100pesos, destaca una tendencia de los comentaristas masculinos a valorar la apariencia física de la muchacha como deseable haciendo un énfasis en su cuerpo (69,6\%); de igual forma, los hombres tienden a aprobar su indumentaria y hacer énfasis en lo corto de su falda $(80 \%)$. Sucede algo parecido en cuanto a la valoración del comportamiento verbal de la protagonista, 34,2\% de los comentaristas hombres celebran sus palabras (Tabla 8), mientras que cuando la valoración se ubica en el ámbito de la moralidad, 42,7 \% de ellos desaprueban su comportamiento (Tabla 9).

Tabla 10. Desaprobación de características de la clase alta.

\begin{tabular}{|lll|}
\hline Vínculo & Frecuencia & $\begin{array}{l}\text { Porcentaje del total de clips } \\
\text { analizados }\end{array}$ \\
Clase social y belleza & 14 & 3.8 \\
Clase social y poder & 43 & $\underline{\mathbf{1 1 . 8}}$ \\
Clase social y liderazgo & 21 & 5.8 \\
Clase social y alta cultura & 14 & 3.8 \\
Clase social e inteligencia & 25 & 6.9 \\
Total de clips analizados & 364 & 100 \\
\hline
\end{tabular}

Elaboración propia.

Ahora bien, para estudiar las prefiguraciones de la clase alta en México que fueron actualizadas en el entramado semántico, se examinaron los clips de video en búsqueda de valoraciones sobre el vínculo entre clase social y distintos aspectos como belleza, poder, liderazgo, alta cultura e inteligencia. Se consideró que los contenidos audiovisuales que proponían una valoración positiva o esperada sobre estos vínculos no ofrecían una propuesta alternativa respecto a la reconfiguración del significado de clase social. En cambio, los clips de video con valoraciones negativas hacia esos vínculos contribuyen a actualizar el significado del concepto de clase alta en México. Respecto de estos vínculos, es necesario precisar que el porcentaje de desaprobación fue muy bajo, solo el vínculo entre clase social y poder fue el que obtuvo un porcentaje de desaprobación relevante (Tabla 10). 


\section{DiSCUSIÓN}

Como se comentó en la sección anterior, hay distintos aspectos que intervienen en el involucramiento activo de las audiencias en YouTube. Con el análisis de la interfaz de este sitio se advirtió que la plataforma aparentemente ofrece una estructura que asegura tanto la diversidad de contenidos como la participación de la audiencia en la construcción de significados de un texto, como en su momento lo indicaron Burgess y Green (2008). No obstante, las funcionalidades de la plataforma no necesariamente logran involucrar a las audiencias de nuevas formas con lo audiovisual. En el caso que hemos estudiado, lo vimos, por ejemplo, en la sección de comentarios donde un porcentaje mínimo de productores participan y dan respuesta a las sugerencias de otros usuarios de YouTube. Esta no parece ser una forma distinta de relacionarnos con lo audiovisual, sino una mediación de la interpretación de textos entre las audiencias de todas partes del mundo. YouTube ofrece el espacio para una comunicación directa entre usuarios y productores de medios, pero eso no significa que en los usos y prácticas sociales se hayan eliminado las barreras de la práctica cultural que los divide. Lo cierto es que la presencia de comentarios de crítica o sugerencia en los videoclips cargados por usuarios activos, youtubers y celebridades parece indicar la existencia de una comunidad de usuarios activa que promueve el involucramiento del público a través de los contenidos que producen.

Por otro lado, en el análisis de la lista de sugerencias de \#lady100pesos, vimos cómo los contenidos creados por las celebridades de YouTube viajan por toda la red del entramado y ocupan un amplio volumen, lo que se traduce en una alta visibilidad y movilidad para dichos contenidos. No obstante, en la gráfica de ruta, se observa cómo el algoritmo de búsqueda del sitio parece concentrar el flujo de las corrientes de significados hacia el centro en donde se ubican youtubers y celebridades del sitio. Esto es importante porque la lista de sugerencias debería asegurar la diversidad de perspectivas y actores al abordar un tema, cosa que no sucede así si los principales actores son siempre los mismos productores semi-profesionales de contenidos. Se enfatiza el calificativo semi-profesional porque a partir de estos datos se podría suponer que los contenidos amateur tienen un amplio espectro de movilidad cuando en realidad se refiere a ese grupo de usuarios que participan dentro del YouTube Partner Program, mismos que indirectamente reciben una remuneración económica por sus producciones audiovisuales y comúnmente ocupan los principales puestos de popularidad, lo que orilla a otros usuarios a buscar alternativas para que sus contenidos tengan mayor audiencia.

Ahora, en cuanto al análisis que se ofrece sobre los contenidos reales de los clips, los resultados parecen indicar que el tipo de producción es más relevante que el formato del video en cuanto a su potencial para involucrar a las audiencias. Como ya se advirtió, el tipo de producción de acción planeada en vivo apareció particularmente asociada con altos niveles de involucramiento, esto podría deberse a que la acción planeada en vivo requiere un nivel de profesionalización por parte del productor que no requieren otros tipos de producción como el recut o el mashup. Si recordamos que en dichos contenidos fue donde más se encontraron referencias de tipo collage, parodia y apropiación, los resultados podrían sugerir que el grado de profesionalización en los productores amateur implica también un involucramiento activo de dichos productores en la crítica al comportamiento de \#lady100pesos. Lo cierto es que, al menos en el caso de estudio que presentamos, los consumidores en YouTube son contundentes al seleccionar contenidos y se involucran activamente con formatos diferentes a los que nos ofrecen los medios tradicionales. Fue así que los newscasts, a pesar de representar un grupo importante de videos, no destacaron en cuanto a niveles de involucramiento; sí destacaron el vlog y el playthorugh, formatos emblemáticos de YouTube.

Aunque la participación en YouTube es limitada, el contenido satírico de los videos y comentarios tiene latente la necesidad de expresar el descontento social. En ese sentido, al examinar los proce-

Ediciones Universidad de Salamanca / CC BY - NC ND Fonseca, Journal of Communication, n. 16, 2018, pp. 155-170 
sos de reconfiguración del caso \#lady100pesos resaltamos la tendencia de los youtubers mexicanos a mantener un sentido moral normativo que podría traducirse en un aspecto negativo si reconocemos que en los comentarios de los hombres persiste una doble moral al referirse al cuerpo de la mujer de manera sexualizada, pero condenando su comportamiento mediante insinuaciones de que la mujer debiera mantener el recato.

No obstante, el sentido moral normativo también puede traducirse en algo positivo si pensamos en la manera en que se censura el acto de corrupción en que intentó incurrir \#lady100pesos: en la muestra analizada observamos una tendencia a desaprobar su comportamiento cívico y, de manera tangencial, desacreditar el poder que ostenta la clase alta en México. Este caso de estudio resultó particularmente relevante porque, como ya se mencionó, es un caso representativo; en los meses posteriores proliferó la documentación en video de casos de corrupción similares a los de \#lady100pesos, algunos de ellos aparecieron en la muestra y fueron categorizados como paratextos. En la posterior popularización de los videos sobre «ladys»o «lords» se puede apreciar una sátira de la lucha de clases en México: las protagonistas de estos videos terminan siendo objeto de burla social por sus aspiraciones a subordinar a otros mediante sobornos o la denigración de los otros actores implicados en el video.

Como estrategia metodológica, el entramado semántico ha sido útil para penetrar en los clústeres de videos que se agrupan en torno a distintos temas y así poder estudiarlos. En YouTube los discursos de la audiencia que asiste a la recepción de un mensaje se multiplican y fortalecen debido a que comparten un mismo espacio en la plataforma mediática, pero sobre todo porque los usuarios de YouTube tienen la capacidad de emitir sus opiniones y comentarios en el mismo código audiovisual que los productores profesionales de medios. Una aproximación cualitativa al contenido de estos videos también es parte del proyecto que dio origen a este artículo y sus resultados se integrarán en comunicaciones futuras. Finalmente, la propuesta de pensar en el texto televisivo como una obra abierta e inacabada que continuamente está siendo recreada (Fiske, 1991) puede extenderse a YouTube toda vez que el mismo sitio reconoce las similitudes que guarda con la televisión en términos de captación de audiencias y de prácticas culturales (YouTube, 2016).

\section{BIBLIOGRAFÍA}

Alexa Internet, Inc. (2017). Top sites in Mexico. Recuperado de Alexa. The web information company: < http://www.alexa.com/topsites/countries/MX $>$.

Burgess, J. y Green, J. (2008). Agency and controversy in the YouTube community. En Proceedings IR 9.0: Rethinking Communities, Rethinking Place-Association of Internet Researchers (AoIR) Conference, IT Universidad de Copenhague, Dinamarca. Recuperado de QUT Digital Repository: $<\underline{\text { http: }: / \text { eprints.qut.edu.au/15383 }}>$.

Burgess, J. y Green, J. (2009). The entrepreneurial vlogger: participatory culture beyond the professional-amateur divide. En P. Snickars y P. Vonderau (eds.), The YouTube reader (pp. 89-107). Estocolmo: National Library of Sweden.

Burgess, J. y Green, J. (2011). YouTube: online video and participatory culture. Cambridge: Polity Press. Fiske, J. (1991). Moments of television: neither the text nor the audience. En E. Seiter, H. Borchers, G. Kreutzner y E. M. Warth (eds.), Remote control. Televison, audiences and cultural power (pp. 56-78). London: Routledge.

Fiske, J. (1992). Audiencing: a cultural studies approach to watching television. Poetics, 21, 345-359. Fiske, J. (1999). Television culture. London: Routledge 
García Canclini, N. (1997). Culturas híbridas y estrategias comunicacionales. Estudios sobre las culturas contemporáneas, 3(5), 109-128.

Google Developers. (2016). YouTube Data API Overview. Recuperado de Google Developers: $<$ https://developers.google.com/youtube/v3/getting-started $>$.

Jenkins, H., Purushotma, R., Clinton, K., Weigel, M. y A. Robinson. (2006). Confronting the challenges of participatory culture: media education for the 21st. century. Iniciativa del programa Building the new field of digital media and learning de la MacArthur Foundation.

Informador. (22 de enero de 2016). Surge 'Lady 100 pesos' en Guanajuato. Recuperado de Informador: < http://www.informador.com.mx/mexico/2016/657317/6/surge-lady-100-pesos-enguanajuato.htm $>$.

Lang, P. (2007). Commenting on comments: investigating responses to antagonism on YouTube. Ponencia presentada en la Society for Applied Anthropology Conference. Tampa, Florida.

Lozano, J. C. (2006). Diversidad cultural y televisión en México. Comunicación y Sociedad, 5, 137-156.

Morris, N. (2002). The myth of unadultered culture meets the threat of imported media. Media, culture and society, 24(2), 278-289.

Muñoz, B. (2012). Negociación de la participación en YouTube. Ponencia presentada en el XXIV Encuentro Nacional de la Asociación Mexicana de Investigadores de la Comunicación. Saltillo, México.

Muñoz, B. (2014). Entamados semánticos: una propuesta para el análisis de la reconfiguración del significado en YouTube. Signo y Pensamiento, 33(64), 16-29.

Muñoz, B. (2016). Narrativa transmedia y paratextualidad en videos amateur en YouTube. Ponencia presentada en el XIII Congreso Latinoamericano de Investigadores de la Comunicación (ALAIC). Cd. de México, México.

Muñoz, B. y Corona, A. (2014). Interacción de videos profesionales y amateur en YouTube. Ponencia presentada en el IV Congreso Internacional de la Asociación Española de Investigación de la Comunicación AE-IC. Bilbao, España.

Muñoz, B. y Vélez, C. (2016). Distribución de videos profesionales y no profesionales en el entramado semántico de YouTube México. Ponencia presentada en el 7. ${ }^{\circ}$ Congreso Internacional de Sociología (UABC). Ensenada, México.

Muñoz, B., Maldonado, L. F. y Vélez, C. (2016). Análisis de la agenda cultural pública en YouTube México. Ponencia presentada en el I Encuentro Regional de la Asociación Mexicana de Investigadores de la Comunicación. Saltillo, México.

Reina, E. (29 de abril de 2016). Bienvenidos al universo 'ladies’ mexicano. Recuperado de El País: <http://elpais.com/elpais/2016/04/28/estilo/1461854120 786112.html>.

Wees, W. (1993). Recycled images: the art and politics of found footage films. New York: Anthology Film Archives.

YouTube. (15 de mayo de 2006). What is YouTube? Recuperado de Internet Archive Way Back Machine: <http://web.archive.org/web/20060515175027/http://youtube.com/t/about>.

YouTube. (2016). Statistics. Recuperado de YouTube: $<$ https://www.youtube.com/yt/press/statistics.html $>$. 